\title{
EOPs \\ Tratamiento del varicocele en la adolescencia
}

\begin{abstract}
Caso clínico
Durante una supervisión, el instructor presenta un caso a los residentes:

un adolescente de 13 años de edad es llevado por su padre a la consulta con su médico de familia por una fimosis. El médico lo examina y decide realizar una consulta al especialista para decidir la conducta a seguir. El paciente regresa semanas más tarde trayendo un informe del urólogo quien sugiere tomar una conducta expectante frente a la fimosis. Sin embargo, le informa que el paciente presenta un varicocele con disminución ligera del tamaño testicular que tiene indicación de cirugía. El padre del niño, algo preocupado, le pregunta al médico de familia si es realmente necesaria la operación, ya que el niño no presenta ninguna molestia. Además, le pide que le informe qué problemas podría ocasionarle a su hijo en el futuro tener un varicocele. El instructor pregunta a los residentes: ¿qué conducta tomarían frente a este paciente?
\end{abstract}

\section{Pregunta que generó el caso}

En un adolescente con varicocele (población), ¿el tratamiento quirúrgico (intervención) previene el daño testicular y la consecuente disminución del potencial fértil (resultado)?

\section{Búsqueda en Medline}

Se emplearon las siguientes palabras clave: "varicocele", "fertility" y "adolescents". La búsqueda fue específica para trabajos de terapéutica empleando el Clinical Queries que ofrece el PubMed. De los trabajos disponibles se seleccionaron aquellos que aportaban mayor grado de evidencia para contestar la pregunta.

\section{¿Qué es el Varicocele?}

El varicocele es una dilatación tortuosa del plexo pampiniforme dentro del cordón espermático. El 90\% se presentan del lado izquierdo, hecho atribuido al trayecto más largo de la vena espermática izquierda que el de su homóloga derecha. La prevalencia de varicocele en la población general se estima cercana al 15\%. Esto ha hecho que muchos autores lo consideren una variante anatómica. El varicocele puede clasificarse clínicamente en tres grados: I. Palpable sólo durante la maniobra de Valsalva; II. Palpable sin la maniobra de Valsalva; y III. Abultamiento visible del escroto. El examen físico debe realizarse con el paciente en posición supina.

La importancia médica de esta entidad tan frecuente radica en su reconocida relación con la infertilidad masculina. Desde el siglo I D.C. en que Celso describía la existencia de hipotrofia testicular en hombres con varicocele, hasta nuestros días, numerosos investigadores han sostenido esta asociación. Entre los argumentos de mayor peso a su favor se destacan: a) la mayor prevalencia de varicocele en las clínicas de infertilidad (20-40\%) en comparación a la reportada en la población general $(15 \%)^{1}$; b) la mejoría de las características del semen y del volumen testicular, luego de la varicocelectomía en pacientes infértiles: ${ }^{2-3}$ y c) la similitud entre el daño producido en modelos de varicocele inducido en animales y el hallado en hombres con varicocele. Varios interrogantes quedan aún por resolver en relación a la historia natural del varicocele y el rol que cumple el mismo en la infertilidad masculina. Entre ellos, se destaca el potencial beneficio de la corrección precoz del varicocele en el adolescente asintomático. Se ha sugerido que el varicocele ejerce un efecto deletéreo progresivo sobre la función testicular. La cirugía precoz ha sido propuesta como intervención con el fin de evitar que se desarrollen alteraciones del espermograma y/o hipotrofia testicular que conduzca de este modo a la infertilidad. De existir evidencia en favor de esta hipótesis debería recomendarse el rastreo del varicocele en los adolescentes asintomáticos. Sin embargo, el papel del varicocele en la fertilidad masculina no parece ser $\tan$ claro. El estudio de la Organización Mundial de la Salud
(WH0 $)^{1}$ muestra que un $85 \%$ de los pacientes con varicocele son fértiles, y un 55\% presentan alguna alteración en el espermograma, indicando que tal relación causal no se cumpliría en todos los casos. La asociación bien podría ser, ni más ni menos que, el mero resultado de dos entidades con una alta prevalencia. Por otro lado, se ha reportado que el consejo y la optimización de la función sexual de la pareja alcanza la misma tasa de fertilidad que la cirugía 4 .

\section{Resumen de los trabajos encontrados}

1) Effect of varicocele treatment in adolescents: a randomized study. Laven et al. Fertil Steril 1992; 58:756-62.

Este estudio se realizó en adolescentes de 17 a 21 años. Se incluyeron 67 pacientes con varicocele, de los cuales 33 fueron asignados al grupo tratamiento (Varicocelectomía) y 34 al grupo sin tratamiento. Además, se incluyó un tercer grupo de 21 adolescentes sanos como grupo control. Al cabo de un año de seguimiento, el grupo tratado alcanzó un volumen testicular similar al del grupo control, y significativamente diferente al del grupo no tratado. En relación al análisis del semen, se halló un incremento significativo de la concentración espermática en el grupo tratado, aunque no necesariamente dicho aumento se correlacionó con un incremento volumen testicular. El estudio concluye que la corrección del varicocele en los adolescentes resulta en un aumento del volumen testicular y de la concentración de semen, aunque no en todos los casos.

2) Repair versus observation in adolescent varicocele: a prospective study.

Paduch D, Niedzielki J. J Urology 1997; 158:1128-1132

Este estudio incluyó adolescentes con varicocele grado II-III de 15 a 19 años. A un grupo de 88 pacientes se les realizó cirugía en tanto que 36 fueron asignados al grupo control. Luego de un año de seguimiento, se realizó una medición ecográfica de los volúmenes testiculares y se calculó el índice de atrofia (\% de reducción en relación al testículo derecho). Se halló que en el grupo tratado había aumentado el volumen testicular y reducido el índice de atrofia en comparación al grupo no tratado con diferencias estadísticamente significativas. No se compararon muestras de semen pre y postoperatorias. El estudio concluye que la cirugía previene la atrofia testicular producida por efecto del varicocele.

\section{3) Sperm parameters after early left varicocele treatment} Lenzi et al. Fertil Steril 1998; 69:347-49

En este trabajo retrospectivo se incluyeron 19 adolescentes que habían sido operados de varicocele entre los 11 y 16 años de edad a los que se les realizó un examen testicular y un análisis del semen 8 años después de la cirugía. Los resultados se compararon con un grupo control de jóvenes no operados (que tenían mismo grado de varicocele que el del grupo tratado antes de ser intervenido), y otro grupo de individuos sanos. En la reexamianción no se detectaron casos de hipotrofia testicular en los grupos con varicocele tratado y no tratado. En relación al análisis del semen, al comparar el grupo tratado con el de jóvenes con varicocele no tratado, se constataron diferencias significativas en la motilidad y formas atípicas, pero no en la concentración espermática. Por el contrario, al comparar el grupo tratado con el de individuos sanos se hallaron diferencias significativas en la concentración espermática, aunque no se observaron cambios en la motilidad de los espermatozoides ni en las formas atípicas. El estudio concluye que la alteración de algunos parámetros seminales asociada al varicocele podrían persistir independientemente de la cirugía, ya que debería esperarse que la misma mejore la concentración espermática en relación al grupo no tratado. Esto podría indicar que las alteraciones del semen se encuentran presentes ya en la niñez, y que estaría asociada con - y no sólo causada por - el varicocele 


\section{Comentario}

La mayoría de los autores coinciden en que el varicocele debe ser operado cuando el mismo molesta o causa dolor, o bien cuando no se logra embarazo luego de 1 año de relaciones sexuales frecuentes y no protegidas (esterilidad). Sin embargo muchos médicos recomiendan la cirugía profiláctica del varicocele en el adolescente asintomático basados en el supuesto de que dicha intervención prevendría el daño testicular y del potencial fértil masculino.

El estudio de Lenzi, con las limitaciones propias de un diseño retrospectivo, encontró un beneficio dudoso en favor de la cirugía. Los otros dos estudios seleccionados presentan un diseño prospectivo randomizado, la mejor evidencia disponible para establecer una relación causa-efecto. Los resultados fueron el volumen testicular y la mejoría de los parámetros del semen. La conclusión de estos estudios es que la varicocelectomía mejoraría los parámetros del semen y el volumen testicular aunque no en todos los casos.

La historia natural del varicocele tiene, como se ha dicho, un final que no es común en todos los casos. Ya se ha mencionado que la alteración en el espermograma no es un fenómeno constante y que la infertilidad se presenta en sólo un $15 \%$ de los pacientes adultos con varicocele. Además, se ha reportado dificultad en establecer un claro deterioro progresivo del espermograma en pacientes con varicocele seguidos en el tiempo ${ }^{5}$. Por otro lado, aunque se ha sostenido que una subpoblación de mayor riesgo sería la de varicoceles de mayor grado y los asociados a hipotrofia testicular, se ha reportado una falta de correlación entre la tasa de fertilidad y la presencia de atrofia testicular en la población adulta 6 . De esta manera, se ha puesto en duda el verdadero valor del tamaño testicular como predictor de infertilidad. Estos hechos, sumados a los reportes de mejoría espontánea del tamaño testicular en adolescentes ${ }^{7}$, arrojan más sombras a la intención de identificar una subpoblación en mayor riesgo que se beneficie de la intervención qurúrgica.

En relación a los parámetos de respuesta a la varicocelectomía, vasta es la literatura médica publicada. La tasa de fertilidad es sin duda el parámetro ideal. Lamentablemente, pocos son los trabajos que la han considerado como resultado principal. La mayoría ha empleado el volumen testicular y las características seminales, los cuales no necesa- riamente guardan una relación directa con la tasa de fertilidad. Por ejemplo, el linfedema resultante podría ser un falso negativo en la detección del índice de atrofia si no se prestan los recaudos adecuados. Independientemente de los resultados considerados, los trabajos han mostrado discrepancias en sus resultados. Varias razones podrían explicar estas diferencias: sesgo de selección* , diferente período de seguimiento, dificultad en definir el grado de varicocele, tamaño de la población, limitaciones propias de los diseños retrospectivos*, entre otras. Para finalizar, un aspecto a considerar es el de los riesgos de la cirugía, los mismos dependen de la técnica y las series consideradas. Con la vía inguinal, la más ampliamente usada, se ha reportado una recurrencia del 4 al 10\% y una incidencia de hidrocele de un 13\%. La escleroterapia, la técnica más sencilla, presenta un riesgo bajo pero de relevancia como es la atrofia testicular.

\section{Conclusiones}

La decisión del tratamiento quirúrgico del varicocele en la población adulta resulta sencilla ya que suele darse en el contexto de infertilidad. En la población adolescente, la indicación no resulta para nada fácil. Sabiendo que una gran mayoría de hombres portadores de varicocele jamás tendrán inconveniente alguno para procrear, sería necesario definir con mayor exactitud el subgrupo de adolescentes en riesgo de infertilidad futura. De otra manera, se estaría exponiendo una gran población de niños sanos, a una cirugía sin un beneficio probado, no exenta de riesgos ni de dificultades técnicas, sin entrar a considerar el trauma psicológico que la misma pudiere ocasionar.

En conclusión, la indicación de varicocelectomía en un adolescente asintomático se basa en un riesgo estadístico mal definido. La cirugía debería considerarse como indicación sólo después de estudiar un gran número de adolescentes, comparando en forma aleatorizada pacientes operados, no operados e individuos sanos en un seguimiento a largo plazo. Esto permitirá identificar los predictores de infertilidad en esta población, y dilucidar finalmente, la historia natural del varicocele.

Por el momento, existe insuficiente evidencia para recomendarse el rastreo del mismo en la población de adolescentes.

*Ver glosario

Dr. Sergio Dominelli

Residente. Unidad de Medicina Familiar y Preventiva. Hospital Italiano de Buenos Aires.

\section{Referencias}

1. WHO Study. The influence of varicocele on paramenters of fertility in a large group of men presenting to infertility clinics. Fertil and Steril 57:12891293, 1992

2. Steckel et al. Relationship between varicocele size and response to varicocelectomy. J Urol 149:769-771, 1993

3. Armand et al. The influence of clinical and subclinical varicocele on testicular volume. Fertil and Steril 68:671-74, 1997.

4. Nieshlag et al. Treatment of Varicocele. Hum Reprod 10:347-53, 1995.

5. Lund and Larsen. A follow-up study of semen quality and fertility in men with varicocele testis and in control subjects. Br J Urol 82:682-686, 1998 .

6. Pinto et al. Varicocele related testicular atrophy and its predictive effect upon fertility. J Urol 152:788-790, 1994.

7. Becmur and Sauvage. Faut-il traiter la varicocele de L'adolescent? Comment? J Chir 136:93-96, 1999. 